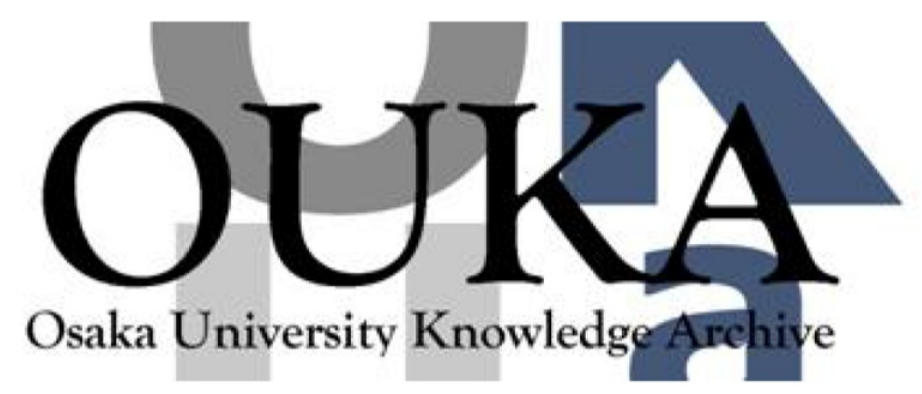

\begin{tabular}{|c|l|}
\hline Title & $\begin{array}{l}\text { Fast ignition relevant study of the flux of } \\
\text { high intensity laser-generated electrons via a } \\
\text { hollow cone into a laser-imploded plasma }\end{array}$ \\
\hline Author(s) & Key, M. H.; Adam, J. C.; Akli, K. U. et al. \\
\hline Citation & Physics of Plasmas. 15(2) p. 022701 \\
\hline Issue Date & $2008-02$ \\
\hline oaire:version & VoR \\
\hline URL & https://hdl. handle. net/11094/3430 \\
\hline rights & \\
\hline Note & \\
\hline
\end{tabular}

Osaka University Knowledge Archive : OUKA

https://ir. Library. osaka-u. ac. jp/

Osaka University 


\title{
Fast ignition relevant study of the flux of high intensity laser-generated electrons via a hollow cone into a laser-imploded plasma
}

\author{
M. H. Key, ${ }^{1}$ J. C. Adam, ${ }^{2}$ K. U. Akli, ${ }^{3}$ M. Borghesi, ${ }^{4}$ M. H. Chen, ${ }^{1}$ R. G. Evans, ${ }^{5}$ \\ R. R. Freeman, ${ }^{6}$ H. Habara, ${ }^{7}$ S. P. Hatchett, ${ }^{1}$ J. M. Hill, ${ }^{1}$ A. Heron, ${ }^{2}$ J. A. King, ${ }^{1}$ \\ R. Kodama, ${ }^{8}$ K. L. Lancaster ${ }^{7}$ A. J. MacKinnon, ${ }^{1}$ P. Patel, ${ }^{1}$ T. Phillips, ${ }^{1}$ L. Romagnani, ${ }^{4}$ \\ R. A. Snavely, ${ }^{1}$ R. Stephens, ${ }^{3}$ C. Stoeckl, ${ }^{9}$ R. Town, ${ }^{1}$ Y. Toyama, ${ }^{8}$ B. Zhang, ${ }^{1}$ \\ M. Zepf ${ }^{4}$ and P. A. Norreys ${ }^{7}$ \\ ${ }^{1}$ University of California, Lawrence Livermore National Laboratory, Livermore, California 94550, USA \\ ${ }^{2}$ Centre de Physique Théorique (UPR14 du CNRS), École Polytechnique, 91128 Palaiseau Cedex, \\ France \\ ${ }^{3}$ General Atomics, San Diego, California 92186, USA \\ ${ }^{4}$ Department of Pure and Applied Physics, Queens University of Belfast, Belfast BT7 1NN, United Kingdom \\ ${ }^{5}$ Blackett Laboratory, Imperial College of Science Technology and Medicine, London SW7 2BZ, \\ United Kingdom \\ ${ }^{6}$ The Ohio State University, Columbus, Ohio 34210, USA \\ ${ }^{7}$ Rutherford Appleton Laboratory, Chilton, Oxon, OX11OQX, United Kingdom \\ ${ }^{8}$ Institute of Laser Engineering, Osaka University, 2-6 Yamada-oka, Suita Osaka 565-0871, Japan \\ ${ }^{9}$ Laboratory for Laser Energetics, University of Rochester, Rochester, New York 14623, USA \\ ${ }^{10}$ University of California, Davis, Department of Applied Science, Livermore, California 94550, USA
}

(Received 19 November 2007; accepted 20 December 2007; published online 15 February 2008)

\begin{abstract}
An integrated experiment relevant to fast ignition . A Cu-doped deuterated polymer spherical shell target with an inserted hollow Au cone is imploded by a six-beam 900-J, 1-ns laser. A 10-ps, 70-J laser pulse is focused into the cone at the time of peak compression. The flux of high-energy electrons through the imploded material is determined from the yield of $\mathrm{Cu} K \alpha$ fluorescence by comparison with a Monte Carlo model. The electrons are estimated to carry about $15 \%$ of the laser energy. Collisional and Ohmic heating are modeled, and Ohmic effects are shown to be relatively unimportant. An electron spectrometer shows significantly greater reduction of the transmitted electron flux than is calculated in the model. Enhanced scattering by instability-induced magnetic fields is suggested. An extension of this fluor-based technique to measurement of coupling efficiency to the ignition hot spot in future larger-scale fast ignition experiments is outlined. () 2008 American Institute of Physics. [DOI: 10.1063/1.2834727]
\end{abstract}

\section{INTRODUCTION}

Compared to conventional "central hot spot" inertial confinement fusion, fast ignition (FI) (Ref. 1) may lead to a higher gain, reduced ignition threshold, and less stringent driver uniformity and target smoothness requirements, with substantially improved prospects for inertial fusion energy. ${ }^{2,3}$ The first integrated FI experiments demonstrated $20 \%$ efficient coupling of 1-ps pulse laser energy to a long-pulse laser imploded plasma using first 60-J (Refs. 4 and 5), and then 350-J (Ref. 6) pulses.

Modeling $^{7}$ shows that for full-scale FI, $>18 \mathrm{~kJ}$ of particle energy injected in $<40 \mu \mathrm{m}$ diameter is required to ignite compressed DT plasma at a density of $300 \mathrm{~g} \mathrm{~cm}^{-3}$. Assuming the reported $20 \%$ coupling efficiency, this implies that $90 \mathrm{~kJ}$ of laser energy would be needed in the short-pulse laser. To generate the required $2-\mathrm{MeV}$ electron energy-the optimum for ignition - the laser pulse must have an appropriate intensity. Constraining the intensity, while supplying the required energy in the required area, leads to pulse durations close to the upper limit which is the inertial confinement time of 10-20 ps, and significantly longer than used in integrated experiments to date. ${ }^{4,6}$

Here we report the first integrated experiment using 10-ps pulses, albeit at much lower intensity than is envisaged for full-scale fast ignition. A spherical shell of $\mathrm{Cu}$-doped deuterated polymer (atomic composition $\mathrm{CD}_{1.4}$ ) with an inserted hollow Au cone was imploded using a powerful nanosecond laser pulse. An intense 10-ps laser pulse was focused into the cone at the time of maximum compression. The electron flux through the implosion was measured from $\mathrm{Cu} K \alpha$ fluorescence. This fluor-based method was first applied to the study of energy transport by laser generated relativistic electrons in planar solid targets. ${ }^{8}$ It makes use of the fact that the cross section for excitation of $K \alpha$ emission by electron collisions varies only weakly above the threshold energy over a multi-MeV range of energies thus providing a good diagnostic of the flux of relativistic electrons. The fluorescence has been selectively imaged using near normal incidence spherical Bragg crystal imagers for which crystals have been found matching $\mathrm{Cu}$ and $\mathrm{Ti} K \alpha$ and the absolute emission has been determined using single hit CCD recording as discussed in this paper. The method has potential for application in future larger-scale integrated experiments because it gives a $K \alpha$ signature predominantly from the region where the product of imploded plasma density and electron flux density is highest. 


\section{THE IMPLODED PLASMA}

Six orthogonal beams from the Vulcan laser ${ }^{9}$ drove the implosion. They had jitter-free synchronization with a chirped pulse amplification (CPA) short pulse, both long and short pulses being derived from the same seed pulse. The implosion drive was $0.9 \mathrm{~kJ}$ in $1 \mathrm{~ns}$ at $1.05 \mu \mathrm{m}$ wavelength, with an approximately Gaussian pulse shape. The beams were focused with $f / 2.5$ lenses with the marginal rays tangential on the microballoon targets. These were unfilled spherical $\mathrm{CD}_{1.4}$ shells doped at 0.7 at. \% with $\mathrm{Cu}$ and typically $486 \mu \mathrm{m}$ in diameter and $6 \mu \mathrm{m}$ thick. An Au hollow cone inserted in the shell was $10 \mu \mathrm{m}$ thick at its tip, which had a 30- $\mu \mathrm{m}$ flat end, located $40 \mu \mathrm{m}$ from the center of the shell. The tapered Au thickness increased to $40 \mu \mathrm{m}$ at the wall of the shell. The cone length was $1 \mathrm{~mm}$ with an exterior full angle of $44^{\circ}$ and an interior full angle of $26^{\circ}$.

Diagnostics directly relevant to this report included a single hit CCD spectrometer recording $\mathrm{Cu} K \alpha$ and an electron spectrometer. The experimental configuration is described more fully elsewhere, together with detailed characterization of the implosion trajectory and density by ps pulse Ti $K \alpha$ radiography ${ }^{10,11}$ and multi-MeV proton radiography. ${ }^{12}$ The key measured implosion parameters were: time of peak compression (3.2 ns after the peak of the drive pulse), compressed plasma diameter $(100 \mu \mathrm{m})$, peak density $\left(4 \mathrm{~g} \mathrm{~cm}^{-3}\right)$, and density $\times$ radius product $\left(20 \mathrm{mg} \mathrm{cm}^{-2}\right)$. Hydrodynamic modeling with the HYDRA code matched to the implosion time, indicated $9 \times 10^{6} \mathrm{~cm} \mathrm{~s}^{-1}$ peak velocity of the implosion and a temperature at stagnation of $75 \mathrm{eV} .^{11}$ These implosion conditions are particularly relevant for FI being close in density and temperature to the cone tip conditions in full-scale FI designs ${ }^{13}$ and, therefore, provide a test bed for possible unstable electron transport at the cone tip.

\section{THE ELECTRON SOURCE CREATED BY THE SHORT-PULSE LASER}

The 10-ps duration short-pulse was focused at $\mathrm{f} / 3$ into the tip of the hollow cone. The average energy on the target was $70 \mathrm{~J}$ with a peak intensity in the vacuum of 3 $\times 10^{18} \mathrm{~W} \mathrm{~cm}^{-2}$. The focal spot had broad wings, and half the power was delivered at an intensity $<10 \%$ of the peak intensity. There was an amplified spontaneous emission (ASE) prepulse of a few ns duration, with an energy approximately $10^{-4}$ of the main pulse energy. This created subcritical density plasma with a scale length at a critical density of about $3 \mu \mathrm{m}$ inferred from two-dimensional hydrodynamic modeling. It is known that self-focusing in the preformed plasma can enhance the laser intensity at the critical density ${ }^{14}$ where most of the absorption occurs, and that absorption at the critical density produces temperatures similar to the ponderomotive energy ${ }^{15}$ for the intensities here, which are well below the level for significant light pressure effects on the temperature. ${ }^{16}$ In addition, electron acceleration processes at subcritical density can generate electrons with temperatures higher than the ponderomotive energy, producing a higher temperature tail on the energy spectrum. Computer modeling suggests that reflection at the walls of the cone can enhance the intensity at its tip and that electrons can be guided along

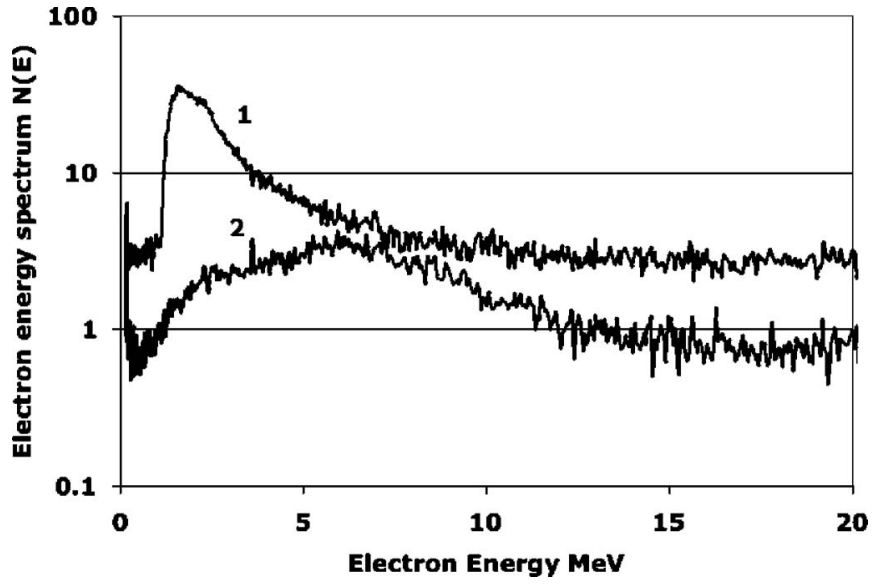

FIG. 1. Electron energy spectra from a free-standing cone (1) and via an implosion (2).

the cone wall to the cone tip. ${ }^{17}$ These rather complex factors together determine the temperature and laser-to-electron energy conversion efficiency of the electron source at the cone tip.

Electron energy spectra along the axis of the CPA beam were measured for electrons escaping from the target using a magnetic deflection spectrometer in vacuum equipped with image plate detection. Details of these measurements have been published earlier. ${ }^{10}$ Spectra are shown in Fig. 1. The temperature of the spectrum is about $1.3 \mathrm{MeV}$ in the 2-6$\mathrm{MeV}$ range for a free-standing cone. It is important to note that the energy spectrum of electrons escaping from the target into vacuum is only indirectly related to that of the generated electrons. A few percent of the electrons escape, the rest is trapped by formation of a Debye sheath at the target surface. Escaping electrons lose energy to the sheath potential. The sheath potential also varies in time. For a fixed sheath potential and a Boltzmann energy spectrum, the escaping electrons have the same temperature as the source electrons. For a two-temperature distribution, the sheath potential may trap the lower-energy electrons so that escaping electrons have a higher temperature.

A multi-temperature distribution is expected, reflecting both the vacuum spatial distribution of intensity, its modification by reflection in the cone, self-focusing and interactions at subcritical density, and changes due to the sheath potential. Consequently, the observed 1.2-MeV slope temperature of the energy spectrum in Fig. 2 is not inconsistent with the $400-\mathrm{keV}$ ponderomotive potential at the 3 $\times 10^{18} \mathrm{~W} \mathrm{~cm}^{-2}$ vacuum peak intensity of the irradiation.

\section{MEASUREMENT OF $K \boldsymbol{\alpha}$ FLUORESCENCE}

The yield of $\mathrm{Cu} K \alpha$ fluorescence was measured with a single hit CCD spectrometer (Spectral Instruments x-ray CCD camera with a $2048 \times 2048$ pixel back-thinned CCD chip with a pixel size of $13-\mu \mathrm{m}$ recorded with 16 -bit resolution) from the rear of the target at an angle of $27^{\circ}$ relative to the CPA beam. The absolute yield was determined from knowledge of the quantum efficiency, the subtended solid angle, and the filter transmission. ${ }^{18}$ The data in Fig. 2 show 


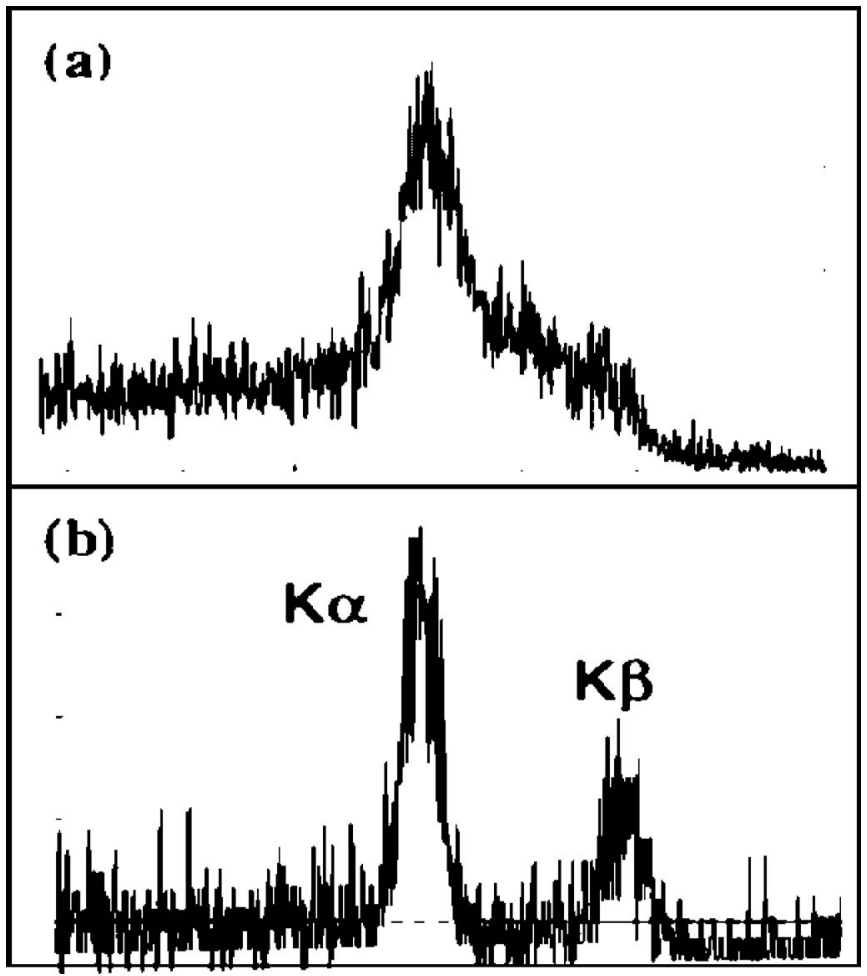

FIG. 2. (a) The single hit CCD Cu $K$ shell fluorescence spectrum from an imploded shell and (b) from a $\mathrm{Cu}$ foil at low energy.

the spectrum from a $20-\mu$ m-thick $\mathrm{Cu}$ foil irradiated at low energy (20 J) exhibiting the expected ratio of $K \alpha$ and $K \beta$ for cold $\mathrm{Cu} K$ shell fluorescence.

The spectrum from a full-energy cone-target implosion with the short pulse timed to coincide with peak compression is also shown in Fig. 2. Here, the $K \alpha$ originates from the electron source generated by the 10-ps pulse and injected via the tip of the cone into the $\mathrm{Cu}$-doped imploded plasma. There might also be a contribution from the nanosecond laser irradiation of the microballoon. Null tests were conducted with long pulse ablation only of the $\mathrm{Cu}$-doped $\mathrm{CD}$ microballoon; no detectable $K \alpha$ yield was observed with the CCD spectrometer. We conclude, therefore, that the spectrum in Fig. 2 is emitted from the short-pulse generated electrons traversing the imploded plasma.

The absence of $K \beta$ gives further support to this interpretation and is attributed to thermal ionization of $\mathrm{M}$ shell electrons in $\mathrm{Cu}$. Multiconfiguration Dirac Fock (MCDF) calculations ${ }^{19}$ were used to compute how $K \beta$ is reduced in intensity relative to $K \alpha$, shifted to higher energy and broadened with increasing ionization. The $75-\mathrm{eV}$ temperature of the imploded plasma at peak compression is sufficient to ionize $\mathrm{Cu}$ to $9+$. The MCDF calculations indicate that for $\mathrm{Cu}^{9+}$, the $K \beta$ line is suppressed relative to $K \alpha$ by an order of magnitude.

The maximum measured absolute yield of $K \alpha$ from an implosion was $1.1 \times 10^{11} \mathrm{~K} \alpha$ photons per steradian.

This understanding is a significant change relative to our earlier preliminary analysis. ${ }^{10}$ In that report, we discussed the puzzling result that data from a Bragg crystal imager gave no detectable image of the $K \alpha$ emission from the implosion

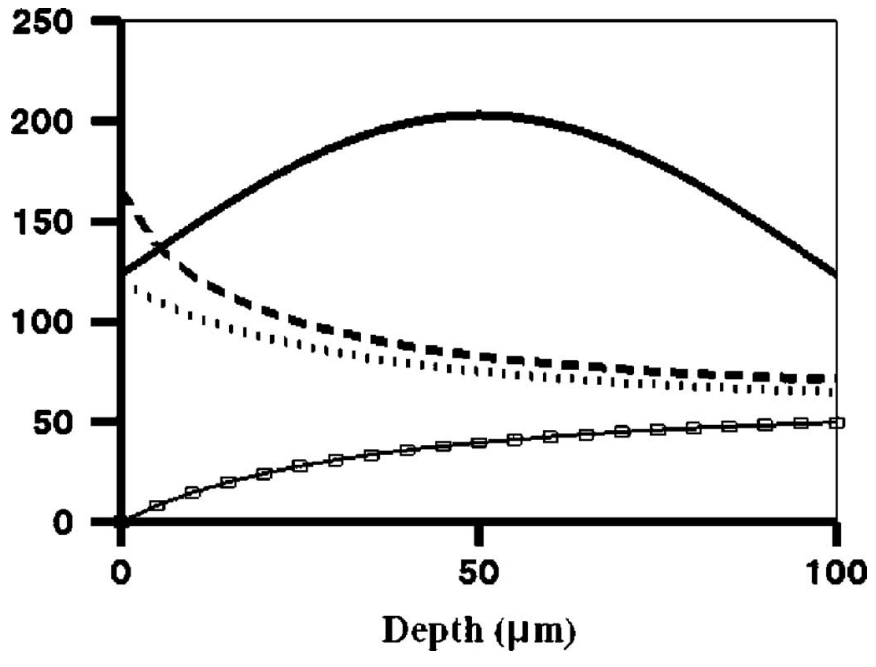

FIG. 3. Heating model: Solid line, density $(\times 50) \mathrm{g} \mathrm{cm}^{-3}$; line with squares, Ohmic potential $(\times 5) \mathrm{keV}$; dashed line, temperature in $\mathrm{eV}$; and dotted line, temperature in $\mathrm{eV}$ with no Ohmic heating.

core. We have since shown that the frequency shift of $K \alpha$ in hot plasma causes major reduction in the reflection efficiency of the extremely narrow band crystal imager, which is consistent with no detectable image of the $K \alpha \cdot{ }^{20}$ Future use of an imager with a bandwidth sufficient to avoid this problem is planned for larger-scale integrated fast ignition experiments.

\section{THE COUPLING EFFICIENCY OF LASER ENERGY TO ENERGY IN ELECTRONS INJECTED INTO THE IMPLOSION}

A key parameter in fast ignition is the coupling efficiency from short-pulse laser energy to the ignition hot spot, and in the present work, the absolute yield of $K \alpha$ is used to infer the flux of electrons injected into the imploded plasma.

To interpret the present data, we applied Monte Carlo modeling with ITS (the integrated Tiger series of coupled Monte Carlo transport codes ${ }^{21}$ ) with various assumed Boltzmann temperatures in the electron source. $10^{6}$ electrons were injected uniformly via a 30- $\mu \mathrm{m}$-source diameter (the tip of the cone) in a $30^{\circ}$ cone angle (similar to experimental observations of the cone angle of electron transport we made earlier $\left.^{8}\right)$, and the injection plane was located $40 \mu \mathrm{m}$ from the center of the density profile in Fig. 3. The $K \alpha$ yield was computed using the published collision cross section, ${ }^{22}$ and the total energy in the electrons was obtained by scaling their number to fit the experimentally measured $K \alpha$ yield. The sensitivity to changes in the assumed source area and cone angle of the electrons in these calculations was found to be slight because of the rather large imploded plasma. Sensitivity to the peak density in the implosion assuming conservation of mass, was such that if the density were higher, the deduced energy in the electrons would be reduced as $\rho^{-2 / 3}$. The greatest sensitivity in these estimates was to the electron temperature as shown in Fig. 4, where the conversion efficiency of laser energy to energy in the electron beam is plotted as a function of the assumed source temperature. 


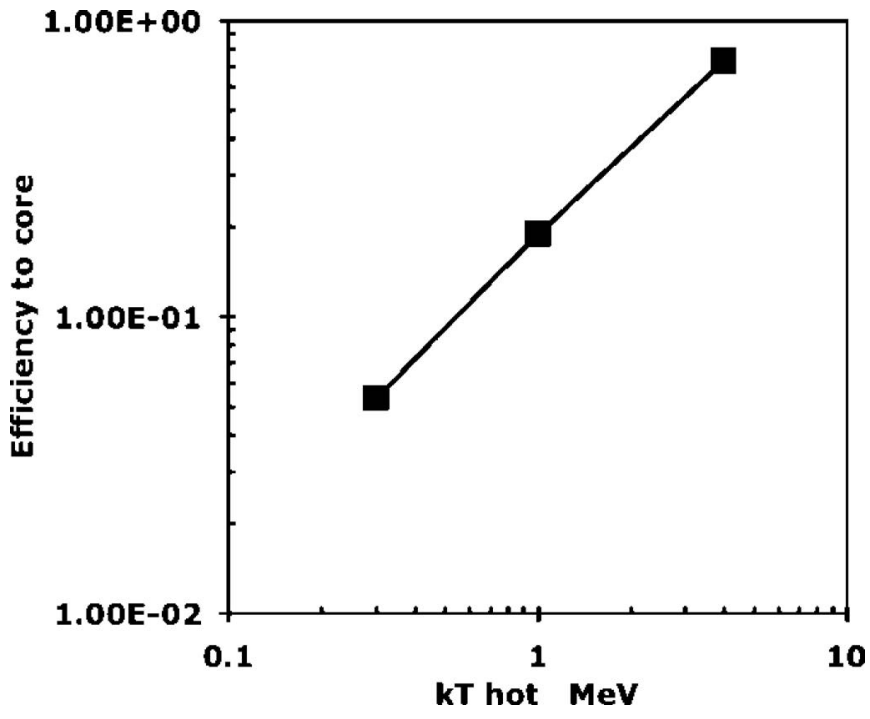

FIG. 4. Conversion fraction of laser energy to energy in electrons traversing the implosion as a function of the temperature of the electrons.

The source temperature, as discussed previously, is a distribution that has an upper bound of $1.2 \mathrm{MeV}$ measured by the electron spectrometer, and at its low end, includes the ponderomotive potential estimate of $0.4 \mathrm{MeV}$. The mean is $0.75 \mathrm{MeV}$, and this temperature, see Fig. 3, implies a conversion efficiency of $15 \%$ of the laser energy to electrons. Taking the previously discussed temperature extremes, a conservative range of uncertainty is from $7 \%$ to $22 \%$.

\section{MODELING OF THE ELECTRON TRANSPORT}

A model was formulated to examine the effects of Ohmic and collisional heating using as an input the typical $15 \%$ of total energy and $0.75-\mathrm{MeV}$ temperature of the electron source. The density profile of the CD was deduced directly from the radiography. The initial temperature and degree of ionization, $75 \mathrm{eV}$ and $Z=4$, respectively, were obtained indirectly from the implosion velocity. The geometry of the electron injection was the same as was used in the Monte Carlo model. Plasma resistivity has its maximum value $^{23}$ at a plateau around $30-50 \mathrm{eV}$, with decreasing resistivity at higher temperatures in the Spitzer regime for $T>100 \mathrm{eV}$. In this model, the resistivity was approximated iteratively as the value at the spatial average temperature and density resulting from the analysis (which was $3 \times 10^{-7} \Omega \mathrm{m}$ from the Sesame tabulation $\left.{ }^{24}\right)$. The energy deposited per unit mass was calculated by injecting electrons in energy bins and calculating their collisional energy loss (using a recent analytic model for electrons in plasma ${ }^{25}$ ) and their Ohmic potential energy, stepping through axial zones along the axis of the energy transport and assuming an equal return current density. The associated collisional and Ohmic heating were also calculated. Temperature rise and degree of ionization were deduced from the Sesame tables. Hydrodynamic and thermal conduction effects were neglected. Figure 3 shows the results.

The temperature maximum was $166 \mathrm{eV}$ located at the entry plane. The absence of implosion core emission in the $\mathrm{x}$-ray pinhole camera images and of measurable thermonuclear neutron yield, put upper bounds of 250 and $750 \mathrm{eV}$, respectively, on the short-pulse heating of the implosion core, which is consistent with the above 166-eV maximum temperature from the transport modeling. The combined Ohmic and collisional heating is greatest at the entry plane because there, the electron flux density is highest and the density is lowest. Ohmic heating contributes only modestly to the temperature rise as evidenced by the plot in Fig. 3 with no Ohmic heating. The $10-\mathrm{keV}$ maximum Ohmic potential is significantly smaller than the $750-\mathrm{keV}$ electron temperature. The relatively small range of deduced temperatures in Fig. 3 makes the approximation of spatially constant resistivity adequate for the estimates made here. The fraction of the injected electron energy absorbed in the target is 9\%, a low value consistent with the small mass per unit area and small Ohmic potential.

\section{DISCUSSION OF THE ENERGY SPECTRA OF ELECTRONS THAT ESCAPE FROM THE TARGET}

In Fig. 1, the data from the electron spectrometer showed that when the imploded plasma was present, there was at least an order of magnitude reduction in the electron signal in the range of $2-5 \mathrm{MeV}$. In most shots there was a greater than 2 order of magnitude reduction to an undetectable level. These data imply either enhanced energy loss or strong scattering. Electron spectrometer data from Kodama et $a l^{4}$ showed a similar but less pronounced reduction. Both effects may be connected with strong excitation of the Weibel-type and two-stream instabilities with associated turbulent magnetic fields. Two-dimensional particle in cell (PIC) modeling has shown that the resulting $B$ fields can strongly scatter the fast electrons. ${ }^{26}$ They can also create a transport barrier by scattering of the return current and generation of an anomalous resistivity and large Ohmic potential. $^{27}$ Using the previously described model of the electron transport, it was determined that 100-fold enhanced resistivity in the model was needed to stop the penetration of most of the electrons, but it raised the temperature in the entry region above $3 \mathrm{keV}$ to a level inconsistent with the experimental absence of thermonuclear neutron and x-ray emission from the heated implosion. Enhanced scattering is therefore a more plausible explanation. Linear analysis ${ }^{28,29}$ gives the growth exponent of the Weibel-type instability, which we estimate at 200 where the electron beam enters the CD plasma. ${ }^{18}$ 2D PIC numerical modeling ${ }^{17}$ for similar injected beam conditions shows strong scattering of the fast electrons. The linear analysis and PIC modeling strongly suggest scattering by instability-generated $B$ fields as the explanation for the reduced signal. This could be an important issue for fast ignition.

\section{Ka AS A DIAGNOSTIC OF COUPLING EFFICIENCY IN FAST IGNITION}

The present work has shown that the total yield of $K \alpha$ can give a measure of the electron flux injected into the implosion. The crucial coupling efficiency in fast ignition is that of laser energy coupled via electron transport to the lim- 
ited spatial region of the ignition hot spot. This can be measured by combining absolutely calibrated spatially crossreferenced imaging of $K \alpha$ fluorescence with short-pulse Compton radiography showing the density structure of the implosion. ${ }^{30}$ Such a method could be used at up to full hydrodynamic scale for high gain FI, but with sub-ignition short-pulse energy, in experiments that are envisaged at new facilities including OMEGA EP, Firex I, and NIF ARC. ${ }^{31}$ Monte Carlo modeling indicates that a pinhole camera with a $K$ edge filter of the same element as the $K \alpha$ fluor would have sufficient selectivity of photon energy to give images dominated by $K \alpha$ but with sufficient bandwidth that the imaging efficiency would be un affected by the temperature-induced frequency shift of $K \alpha$. The photon energy of the fluor should be high enough for escape from the compressed plasma and for brightness of fluorescence exceeding the background from thermal emission from the plasma. Ag $K \alpha$ appears suitable on both counts. Tests of filtered pinhole camera imaging of $\mathrm{Ag} K \alpha$ will be reported in a future publication, and in the longer term, the method described here is envisaged as a key diagnostic of coupling efficiency in larger-scale integrated fast ignition experiments.

\section{CONCLUSIONS}

In conclusion, we have made the first measurement of electron energy transport via an imploded plasma in a FI cone-inserted target using a 10-ps pulse duration similar to that required for full-scale experiments. We have demonstrated a new $K \alpha$ fluorescence diagnostic to determine the efficiency of converting laser energy to electron energy injected into an implosion. The results suggest that for the conditions of this experiment, about $15 \%$ of the laser energy is converted to electron energy traversing the implosion. The data also show an unexpectedly strong reduction of the electron flux density transmitted through the imploded plasma and recorded in the vacuum, relative to that measured from the cone without an implosion. It is concluded on the basis of PIC modeling and linear instability theory that this is best explained by scattering from strong $B$ fields generated by Weibel-type filamentation instability occurring where the electrons leave the cone tip and enter the imploded plasma. A scheme has been outlined for extending the $K \alpha$ diagnostic method to measure the coupling efficiency between shortpulse laser energy and the ignition hot spot in future largerscale integrated fast ignition experiments.

\section{ACKNOWLEDGMENTS}

We thank R. Petrasso for advice on electron energy loss in plasma, and we are grateful to the staff of the Central Laser Facility, in particular J. Collier, S. Hawkes, R. Heathcote, C. Hernandez-Gomez, D. Neely, and M. H. R. Hutchinson, for their support.

This work was performed under the auspices of the U.S. Department of Energy by the Lawrence Livermore National Laboratory under Contract No. W-7405-ENG-48 and with grant support (GR/R16778) from the U.K. EPSRC. M.Z. thanks the Royal Society for a Wolfson research merit award.

${ }^{1}$ N. G. Basov, S. Y. Guskov, and L. P. Feokistov, J. Sov. Laser Res. 13, 396 (1992); M. Tabak, J. Hammer, M. E. Glinsky, W. L. Kruer, S. C. Wilks, J. Woodworth, E. M. Campbell, and M. D. Perry, Phys. Plasmas 1, 1626 (1994).

${ }^{2}$ M. H. Key, E. M. Campbell, T. E. Cowan et al., J. Fusion Energy 17, 231 (1998).

${ }^{3}$ M. H. Key, Phys. Plasmas 14, 055502 (2007).

${ }^{4}$ R. Kodama, P. A. Norreys, K. Mima et al., Nature (London) 412, 798 (2001).

${ }^{5}$ M. H. Key, Nature (London) 412, 775 (2001).

${ }^{6}$ R. Kodama, H. Shiraga, K. Shigemori et al., Nature (London) 418, 933 (2002).

${ }^{7}$ S. Atzeni, Phys. Plasmas 6, 3316 (1999).

${ }^{8}$ K. B. Wharton, S. P. Hatchett, S. C. Wilks, M. H. Key, J. D. Moody, V. Yanovsky, A. A. Offenberger, B. A. Hammel, M. D. Perry, and C. Joshi, Phys. Rev. Lett. 81, 822 (1998); R. B. Stephens, R. A. Snavely, Y. Aglitski et al., Phys. Rev. E 69, 066414 (2004).

${ }^{9}$ C. N. Danson, J. Collier, D. Neely et al., J. Mod. Opt. 45, 1653 (1998).

${ }^{10}$ P. A. Norreys, K. L. Lancaster, C. D. Murphy et al., Phys. Plasmas 11, 2746 (2004).

${ }^{11}$ J. A. King, K. Akli, B. Zhang et al., Appl. Phys. Lett. 86, 191501 (2005).

${ }^{12}$ A. J. MacKinnon, P. K. Patel, R. P. Town et al., Rev. Sci. Instrum. 75, 3531 (2004); A. J. MacKinnon, P. K. Patel, M. Borghesi et al., Phys. Rev. Lett. 97, 045001 (2006).

${ }^{13}$ S. P. Hatchett, D. Clark, M. Tabak, R. E. Turner, C. Stoeckl, R. B. Stephens, H. Shiraga, and K. Tanaka, Fusion Sci. Technol. 49, 327 (2006).

${ }^{14}$ D. E. Hinkel, E. A. Williams, R. L. Berger, L. V. Powers, A. B. Langdon, and C. H. Still, Phys. Plasmas 5, 1887 (1998).

${ }^{15}$ S. C. Wilks, W. L. Kruer, M. Tabak, and A. B. Langdon, Phys. Rev. Lett. 69, 1383 (1992).

${ }^{16}$ S. C. Wilks, Phys. Fluids B 5, 2603 (1993); E. Lefebvre and G. Bonnaud, Phys. Rev. E 55, 1011 (1997).

${ }^{17}$ Y. Sentoku, K. Mima, H. Ruhl, Y. Toyama, R. Kodama, and T. E. Cowan, Phys. Plasmas 11, 3083 (2004).

${ }^{18}$ C. Stoeckl, W. Theobald, and T. C. Sangster, Rev. Sci. Instrum. 75, 3705 (2004).

${ }^{19}$ M. H. Chen, Phys. Rev. A 31, 1449 (1985).

${ }^{20}$ K. U. Akli, M. H. Key, H. K. Chung et al., Phys. Plasmas 14, 023102 (2007).

${ }^{21}$ J. A. Halbeib and T. A. Mehlhorn, Sandia National Laboratory Report No. SAND 84-0573 (1984). See National Technical Information Service Document No. DE85003719, The Integrated TIGER Series of Coupled Electron/Photon Monte Carlo Transport Codes. Copies may be ordered from the National Technical Information Service, Springfield, VA 22161.

${ }^{22}$ H. Deutsch, P. Scheier, K. Becker, and T. D. Mark, Int. J. Mass. Spectrom. 233, 13 (2004).

${ }^{23}$ H. M. Milchberg, R. R. Freeman, and S. C. Davey, Phys. Rev. Lett. 62, 2364 (1988); Y. T. Lee and R. M. More, Phys. Fluids 27, 1273 (1984).

${ }^{24}$ S. P. Lyon and J. D. Johnson, Los Alamos National Laboratory Report No. LA-UR-92-3407. SESAME: the Los Alamos National Laboratory equation of state database (1992), available online at http://lib-www.lanl.gov/ cgi-bin/getfile?00612717.pdf

${ }^{25}$ C. K. Li and R. D. Petrasso, Phys. Rev. E 70, 067401 (2004); C. Deutsch, H. Furukawa, K. Mima, M. Murakami, and K. Nishihara, Phys. Rev. Lett. 77, 2483 (1996)

${ }^{26}$ J. C. Adam, A. Heron, and G. Laval, Phys. Rev. Lett. 97, 205006 (2007).

${ }^{27}$ Y. Sentoku, K. Mima, P. Kaw, and K. Nishikawa, Phys. Rev. Lett. 90, 155001 (2003).

${ }^{28}$ J. M. Hill, M. H. Key, S. P. Hatchett, and R. Freeman, Phys. Plasmas 12, 082304 (2005)

${ }^{29}$ C. Deutsch, A. Bret, M.-C. Firpo, and P. Fromy, Phys. Rev. E 72, 026402 (2005).

${ }^{30}$ R. Tommasini, H.-S. Park, P. Patel et al., Atomic Processes in Plasmas, CP926, edited by J. D. Gillaspy, J. J. Curry, and W. L. Wiese (American Institute of Physics, New York, 2007), 978-0-7354-0436-6/07, pp. 248-255.

${ }^{31}$ C. P. J. Barty, M. H. Key, J. Britten et al., Nucl. Fusion 44, 256 (2004). 